\title{
Parity indices of partitions
}

\author{
by \\ KAĞAN KurŞUnGÖZ (University Park, PA)
}

1. Introduction. In A2, Andrews made an extensive study of parity in partitions. Roughly the second half of his paper $(\S \S 6-11)$ is devoted to parity indices of partitions. The generating functions which classify partitions subject to various constraints according to their parity indices are given. The main method Andrews employs is to solve functional equations that derive from the definitions of parity indices. Here, we give purely combinatorial constructions of those generating functions. We also prove some relations between them either bijectively or sieve-theoretically.

A partition $\lambda$ of a positive integer $n$ is a nonincreasing sequence of positive integers $\lambda_{1} \geq \cdots \geq \lambda_{k}>0$ such that $n=\lambda_{1}+\cdots+\lambda_{k}$ [A1, Ch. 1]. A pictorial representation for a partition is its Ferrers graph, a left justified table of dots such that the first row has $\lambda_{1}$ dots and so on. For instance, $36=9+7+7+5+4+2+2$ has the following Ferrers graph:

The conjugate $\lambda^{\prime}$ of a partition $\lambda$ is obtained by reflecting the Ferrers graph across the main diagonal. For the partition above, the conjugate is

2010 Mathematics Subject Classification: Primary 05A15, 05A17, 05A19; Secondary $11 \mathrm{P} 81$.

Key words and phrases: integer partition, parity index, generating function. 
$7+7+5+5+4+3+3+1+1$, and has the following Ferrers graph:

Generating functions for most classes of partitions require heavy use of $q$-factorials, which are defined by

$$
(a ; q)_{n}=\prod_{i=1}^{n}\left(1-a q^{i-1}\right), \quad(a ; q)_{\infty}=\lim _{n \rightarrow \infty}(a ; q)_{n}=\prod_{i=1}^{\infty}\left(1-a q^{i-1}\right) .
$$

If the base is no other than $q$, we write $(a)_{n}=(a ; q)_{n}$ [A1, §2.2].

We also need to recall the definition of Gaussian polynomials [A1, §3.3] before we proceed:

$$
\left[\begin{array}{l}
A \\
B
\end{array}\right]_{r}=\frac{\left(q^{r} ; q^{r}\right)_{A}}{\left(q^{r} ; q^{r}\right)_{B}\left(q^{r} ; q^{r}\right)_{A-B}},
$$

for $0 \leq B \leq A$, and $r$ a non-zero integer. If $r=1$, then the base can be omitted, and we write

$$
\left[\begin{array}{l}
A \\
B
\end{array}\right]=\left[\begin{array}{l}
A \\
B
\end{array}\right]_{1}
$$

We will be using the fact that $\left[\begin{array}{l}A \\ B\end{array}\right]$ generates partitions into at most $B$ parts, all $\leq A-B$ [A1, Theorem 3.1].

2. Basic constructions. Throughout the paper, for a fixed positive integer $n$, let $\lambda=\left(\lambda_{1}, \ldots, \lambda_{n}\right)$ be a partition such that $\lambda_{1} \geq \cdots \geq \lambda_{n}$. Depending on context, we will impose certain restrictions on the parity of parts of $\lambda$. Also, we let $\mu=\left(\mu_{1}, \ldots, \mu_{j}\right)$ be a partition consisting of distinct numbers such that $n \geq \mu_{1}>\cdots>\mu_{j}>0$. Let $\mu^{\prime}=\left(\mu_{1}^{\prime}, \ldots, \mu_{r}^{\prime}\right)$ be the conjugate partition of $\mu$. It follows that $j \leq n, \mu_{1}^{\prime}=j, \mu_{r}^{\prime}=1$, and that $\mu^{\prime}$ contains instances of every integer from 1 to $j$.

2.1. Lower parity indices in partitions with distinct parts. We begin with a definition from A2. 
Definition 2.1. The lower even (resp. odd) parity index of a partition $\lambda$ is the length of the longest subsequence of parts in $\lambda$ that alternate in parity, where the smallest element in the subsequence is even (resp. odd). It is zero if all parts are odd (resp. even).

It is clear that the lower even parity index of a partition is the number of times the parity changes from the smallest part to the largest, beginning with an even part, plus one for that smallest even element (likewise for the lower odd parity index).

ExAmple. $\widetilde{\lambda}=(15,13,12,8,5,4)$ has lower even parity index 4 , since beginning with the smallest even part (4), and considering the greater parts in their increasing order, there are parity changes at 5,8 , and 13 . Also, $\tilde{\lambda}$ has lower odd parity index 3 .

TheOREM 2.2. Let $p_{o}(r, m, n)$ (respectively, $p_{e}(r, m, n)$ ) denote the number of partitions of $n$ into $m$ distinct parts with lower odd (respectively, even) parity index equal to $r$. Then

$$
\begin{aligned}
P_{o}(y, x ; q) & =\sum_{m, n, r \geq 0} p_{o}(r, m, n) x^{m} y^{r} q^{n} \\
& =1+\sum_{n=1}^{\infty} \frac{x^{n} q^{n(n+1)}(-y / q ; 1 / q)_{n}}{\left(q^{2} ; q^{2}\right)_{n}} .
\end{aligned}
$$

This is [A2, Theorem 7, eq. (7.4)].

Proof. Let $\lambda$ have distinct even parts. Let $\widetilde{\lambda}=\left(\widetilde{\lambda}_{1}, \ldots, \widetilde{\lambda}_{n}\right)$ be such that

$$
\begin{aligned}
\widetilde{\lambda}_{1} & =\lambda_{1}-\mu_{1}^{\prime}=\lambda_{1}-j, \\
& \vdots \\
\widetilde{\lambda}_{r} & =\lambda_{r}-\mu_{r}^{\prime}=\lambda_{r}-1, \\
\widetilde{\lambda}_{r+1} & =\lambda_{r+1}, \\
& \vdots \\
\widetilde{\lambda}_{n} & =\lambda_{n} .
\end{aligned}
$$

Then the parities of parts of $\tilde{\lambda}$ counted from the smallest look like

$$
E, \ldots, E, \underbrace{O, \ldots, O}_{1 \text { subtracted }}, \underbrace{E, \ldots, E}_{\text {subtracted }}, \ldots, \underbrace{(O / E), \ldots,(O / E)}_{j \text { subtracted }} .
$$

so that the lower odd parity index of $\widetilde{\lambda}$ is $j$, and the parts of $\widetilde{\lambda}$ are still distinct, since from two adjacent even parts, either the same numbers, or a consecutive pair of numbers, in their respective order, are subtracted. Moreover, either 1 or 0 is subtracted from the first part, so $\widetilde{\lambda}$, too, has $n$ 
parts. Note that, in comparison to $\$ 2.2$, adding parts of $\mu^{\prime}$ to parts of $\lambda$ does not serve the purpose. Doing so would introduce leaps of at least 3 when the parity is switched.

Conversely, given $\widetilde{\lambda}$, a partition into distinct parts with lower odd parity index $j$, we can add 1 to the first subsequence of adjacent odd parts, 2 to the following subsequence of adjacent even parts and so on to recover a partition $\lambda$ with distinct even parts, and $\mu^{\prime}$ whose conjugate is a partition into $j$ distinct numbers $\leq n$.

The result follows, since $q^{n(n+1)} /\left(q^{2} ; q^{2}\right)_{n}$ generates partitions into distinct even parts $(\lambda)$; and $(-y / q ; 1 / q)_{n}$ generates the partitions into distinct parts, all $\leq n(\mu)$. The base is $1 / q$ instead of $q$ in the latter factor because we are subtracting. Each $y$ indicates a parity change, the first to an odd number. Thus, the exponent of $y$ keeps track of the lower odd parity index.

If we switch the base to $q$ in $(-y / q ; 1 / q)_{n}$ GR, eq. (1.2.24)], then [A2, eq. (7.4)] follows.

There is an alternative way to explain [A2, eq. (7.4)] in its original form. $x^{n} y^{n} q^{n(n+1) / 2} /\left(q^{2} ; q^{2}\right)_{n}$ generates partitions with the largest possible lower odd parity index. Then for each factor in $(-q / y)_{n}$, say $\left(1+q^{j} / y\right)(1 \leq j \leq n)$, 1 leaves the partition intact, and $q^{j} / y$ adds 1 to the first $j$ parts, ruling out a parity change at the $j$ th part.

Example. Let $\lambda=(18,16,14,10,6,4)$ and $\mu=(5,4,2)$. Then $\mu^{\prime}=$ $(3,3,2,2,1)$, so that $\tilde{\lambda}=(15,13,12,8,5,4)$. The lower parity index of $\tilde{\lambda}$ is 3 . The Ferrers graphs of the partitions involved are the following (in the second $\lambda$, the $\times$ 's indicate $\mu^{\prime}$, which has to be deleted in order to obtain $\widetilde{\lambda}$ ): 


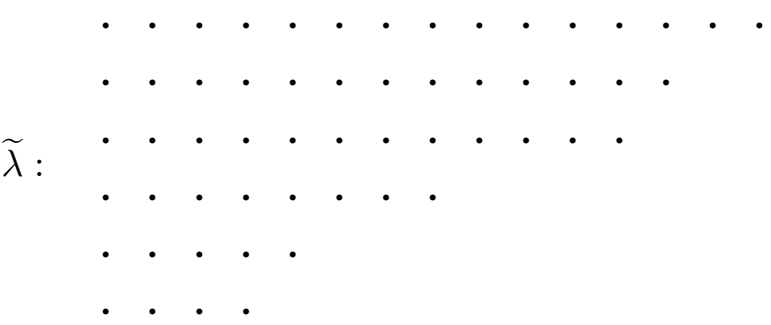

The following equation gives the relation between $P_{e}(y, x ; q)$ and $P_{o}(y, x q ; q)$ :

$$
P_{e}(y, x ; q)=(1+x q) P_{o}(y, x q ; q) .
$$

How to obtain (2.3) [A2, eq. (7.3)] is already explained combinatorially in that paper.

\subsection{Lower parity indices in unrestricted partitions}

TheOREM 2.3. Let $u_{e}(r, m, n)$ (respectively, $u_{o}(r, m, n)$ ) denote the number of partitions of $n$ into $m$ parts with lower even (respectively, odd) parity index equal to $r$. Then

$$
U_{e}(y, x ; q)=\sum_{m, n, r \geq 0} u_{e}(r, m, n) x^{m} y^{r} q^{n}=1+\sum_{n=1}^{\infty} \frac{x^{n} q^{n}(-y q ; q)_{n}}{\left(q^{2} ; q^{2}\right)_{n}} .
$$

Proof. Suppose that $\lambda$ has all odd parts. Let $\widetilde{\lambda}=\left(\widetilde{\lambda}_{1}, \ldots, \widetilde{\lambda}_{n}\right)$ be such that

$$
\begin{aligned}
\widetilde{\lambda}_{1} & =\lambda_{1}+\mu_{1}^{\prime}=\lambda_{1}+j, \\
& \vdots \\
\widetilde{\lambda}_{r} & =\lambda_{r}+\mu_{r}^{\prime}=\lambda_{r}+1, \\
\widetilde{\lambda}_{r+1} & =\lambda_{r+1}, \\
& \vdots \\
\widetilde{\lambda}_{n} & =\lambda_{n} .
\end{aligned}
$$

Then the parities of parts of $\tilde{\lambda}$ counted from the smallest look like

$$
O, \ldots, O, \underbrace{E, \ldots, E}_{1 \text { added }}, \underbrace{O, \ldots, O}_{2 \text { added }}, \ldots, \underbrace{(E / O), \ldots,(E / O)}_{j \text { added }},
$$

so that the lower even parity index of $\widetilde{\lambda}$ is $j$.

Note that, conversely, given a partition $\widetilde{\lambda}$ with lower even parity index $j$, we can subtract 1 from the first subsequence of adjacent even numbers, 2 from the following sequence of adjacent odd numbers, and so on, to obtain 
a partition $\lambda$ into odd numbers, and a partition $\mu^{\prime}$ in which all parts between 1 and $j$ appear. Moreover, $\widetilde{\lambda}$ and $\lambda$ are related as in 2.5.

This constructs the generating function, since $q^{n} /\left(q^{2} ; q^{2}\right)_{n}$ generates partitions into odd parts $(\lambda) ;(-y q ; q)_{n}$ generates partitions into distinct parts, all $\leq n(\mu)$; and the exponent of $y$ is $j=r$ above, which is the lower even parity index as well as the number of parts in $\mu$.

ExAMPLE. We will work backwards in this example. That is, given $\tilde{\lambda}$ with a certain lower even parity index, we will find a partition $\lambda$ into odd parts, and a partition $\mu$ with distinct parts.

Let $\widetilde{\lambda}=(8,5,5,4,2,1,1)$. The lower even parity index of $\widetilde{\lambda}$ is 3 . By the above discussion, when we group the adjacent parts that have the same parity, we have

$$
1,1, \underbrace{2,4}_{1 \text { added }}, \underbrace{5,5}_{2 \text { added }}, \underbrace{8}_{3 \text { added }} .
$$

So, $\mu^{\prime}=(3,2,2,1,1), \mu=(5,3,1)$, and $\lambda=(5,3,3,3,1,1,1)$, as desired.

In the above construction, if we replace $x$ by $x / q$, then we will have converted the lower even parity index into the lower odd parity index. But then partitions may include zeros counted by $x$ 's. If we multiply by $1-x$ at the front, then we will have eliminated those unwanted partitions. It follows that

$$
\begin{aligned}
&(1-x)\left(1+\sum_{n=1}^{\infty} \frac{x^{n}(-y q ; q)_{n}}{\left(q^{2} ; q^{2}\right)_{n}}\right) \\
&=\sum_{m, n, r \geq 0} u_{o}(r, m, n) x^{m} y^{r} q^{n}=U_{o}(y, x ; q) .
\end{aligned}
$$

This is [A2, eq. (8.5)].

Similar to (2.3), there is a nice relation between $U_{e}(y, x ; q)$ and $U_{o}(y, x ; q)$ given by

$$
U_{e}(y, x ; q)=\frac{U_{o}(y, x ; q)}{1-x q} .
$$

This is [A2, eq. (8.3)], and the derivation is explained combinatorially in that paper.

2.3. Upper parity indices in unrestricted partitions. We recall one more definition from $\mathrm{A} 2$.

Definition 2.4. The upper even (resp. odd) parity index of a partition $\lambda$ is the length of the longest subsequence of parts in $\lambda$ that alternate in parity, where the largest element is even (resp. odd). It is zero if all parts are odd (resp. even). 
It is easily seen that the upper even parity index equals the number of times parts of $\lambda$ change parity from the largest to the smallest, beginning with an even one, plus one for that largest even element (similarly for the upper odd parity index).

ExAmple. $\widetilde{\lambda}=(15,13,12,8,5,4)$ has upper even parity index 3 , since beginning with the greatest even part (12), and considering the smaller parts in their decreasing order, there are parity changes at 5 , and 4 . Also, $\widetilde{\lambda}$ has upper odd parity index 4 .

In [A2, $\S 10]$, the following generating functions are defined:

$$
\begin{aligned}
& F_{e}(N, y, x ; q):=F_{e}(N)=\sum_{r, m, n \geq 0} \phi_{e}(N, r, m, n) y^{r} x^{m} q^{n}, \\
& F_{o}(N, y, x ; q):=F_{o}(N)=\sum_{r, m, n \geq 0} \phi_{o}(N, r, m, n) y^{r} x^{m} q^{n},
\end{aligned}
$$

where $\phi_{e}(N, r, m, n)$ (respectively, $\left.\phi_{o}(N, r, m, n)\right)$ is the number of partitions of $n$ into $m$ parts each at most $N$, with upper even (respectively, upper odd) parity index equal to $r$ ([A2, eqs. (10.1) and (10.2)]).

It is clear that upon $N \rightarrow \infty, F_{e}(\infty)$ (respectively, $F_{o}(\infty)$ ) generates partitions into unrestricted and unbounded parts classified according to their upper even (respectively, odd) parity indices.

From this point onwards, we require that $\mu$ has distinct parts $<n$ (but not $\leq n$ as in 2.1 and $\$ 2.2$.

THEOREM 2.5 .

$$
\begin{aligned}
F_{e}(\infty)= & \sum_{j, n \geq 0} \frac{x^{n} y^{2 j+1} q^{2 j(2 j+1) / 2+2 n}}{\left(q^{2} ; q^{2}\right)_{n}}\left[\begin{array}{c}
n \\
2 j+1
\end{array}\right] \\
& +\sum_{j, n \geq 0} \frac{x^{n} y^{2 j} q^{(2 j-1) 2 j / 2+n}}{\left(q^{2} ; q^{2}\right)_{n}}\left[\begin{array}{c}
n \\
2 j
\end{array}\right] .
\end{aligned}
$$

This is [A2, eq. (10.15)].

Proof. Given a partition $\lambda$ with even parts, if $\tilde{\lambda}$ is constructed as in 2.5), then for $\mu$ having $2 j$ parts, the parities of parts of $\tilde{\lambda}$ beginning with the smallest look like

$$
E, \ldots, E, \underbrace{O, \ldots, O}_{1 \text { added }}, \ldots, \underbrace{O, \ldots, O}_{2 j-1 \text { added }}, \underbrace{E, \ldots, E}_{2 j \text { added }}
$$

and for $\mu$ having $2 j+1$ parts, the parities of parts of $\tilde{\lambda}$ beginning with the smallest look like

$$
E, \ldots, E, \underbrace{O, \ldots, O}_{1 \text { added }}, \ldots, \underbrace{E, \ldots, E}_{2 j \text { added }}, \underbrace{O, \ldots, O}_{2 j+1 \text { added }}
$$


where both options have upper even parity index $2 j$. Now, the partitions with exactly $2 j$ (respectively $2 j+1$ ) distinct parts, all $\leq n-1$, are generated by

$$
q^{2 j(2 j+1) / 2}\left[\begin{array}{c}
n-1 \\
2 j
\end{array}\right] \quad\left(\text { respectively, } q^{(2 j+1)(2 j+2) / 2}\left[\begin{array}{c}
n-1 \\
2 j+1
\end{array}\right]\right) .
$$

On the other hand, given a partition $\lambda$ with odd parts, if $\widetilde{\lambda}$ is constructed as in 2.5), then for $\mu$ having $2 j$ parts, the parities of parts of $\tilde{\lambda}$ beginning with the smallest look like

$$
O, \ldots, O, \underbrace{E, \ldots, E}_{1 \text { added }}, \ldots, \underbrace{E, \ldots, E}_{2 j-1 \text { added }}, \underbrace{O, \ldots, O}_{2 j \text { added }},
$$

and for $\mu$ having $2 j-1$ parts, the parities of parts of $\tilde{\lambda}$ beginning with the smallest look like

$$
O, \ldots, O, \underbrace{E, \ldots, E}_{1 \text { added }}, \ldots, \underbrace{O, \ldots, O}_{2 j-2 \text { added }}, \underbrace{E, \ldots, E}_{2 j-1 \text { added }},
$$

where both options have upper even parity index $2 j$. Now, the partitions with exactly $2 j$ (respectively $2 j-1$ ) distinct parts, all $\leq n-1$, are generated by

$$
q^{2 j(2 j+1) / 2}\left[\begin{array}{c}
n-1 \\
2 j
\end{array}\right] \quad\left(\text { respectively, } q^{(2 j-1) 2 j / 2}\left[\begin{array}{c}
n-1 \\
2 j-1
\end{array}\right]\right) .
$$

Observe that given $\widetilde{\lambda}$, we can form $\lambda$ and $\mu$, and the procedures are inverses of each other, as in 2.5.)

Combining these computations in the light of the arguments employed above, namely adding parts of $\mu^{\prime}$ to parts of $\lambda$, we get

$$
\begin{aligned}
& F_{e}(\infty)= \\
& \sum_{n \geq 0} \frac{x^{n} q^{2 n}}{\left(q^{2} ; q^{2}\right)_{n}}\left\{\sum_{j}\left(q^{2 j(2 j+1) / 2}\left[\begin{array}{c}
n-1 \\
2 j
\end{array}\right]+q^{(2 j+1)(2 j+2) / 2}\left[\begin{array}{c}
n-1 \\
2 j+1
\end{array}\right]\right) y^{2 j+1}\right\} \\
& +\sum_{n \geq 0} \frac{x^{n} q^{n}}{\left(q^{2} ; q^{2}\right)_{n}}\left\{\sum_{j}\left(q^{2 j(2 j+1) / 2}\left[\begin{array}{c}
n-1 \\
2 j
\end{array}\right]+q^{(2 j-1) 2 j / 2}\left[\begin{array}{c}
n-1 \\
2 j-1
\end{array}\right]\right) y^{2 j}\right\} \\
& =\sum_{j, n \geq 0} \frac{x^{n} y^{2 j+1} q^{2 j(2 j+1) / 2+2 n}}{\left(q^{2} ; q^{2}\right)_{n}}\left[\begin{array}{c}
n \\
2 j+1
\end{array}\right]+\sum_{j, n \geq 0} \frac{x^{n} y^{2 j} q^{(2 j-1) 2 j / 2+n}}{\left(q^{2} ; q^{2}\right)_{n}}\left[\begin{array}{c}
n \\
2 j
\end{array}\right] \text {, }
\end{aligned}
$$

by [A1, p. 35, eq. (3.3.4)]. 
THEOREM 2.6.

$$
\begin{aligned}
& F_{e}(2 M+1) \\
& =\sum_{j, n \geq 0} x^{n} y^{2 j+1} q^{2 j(2 j+1) / 2+2 n}\left[\begin{array}{c}
M-j+n-1 \\
n
\end{array}\right]_{2}\left[\begin{array}{c}
n \\
2 j+1
\end{array}\right] \\
& \quad+\sum_{j, n \geq 0} x^{n} y^{2 j} q^{(2 j-1) 2 j / 2+n}\left[\begin{array}{c}
M-j+n \\
n
\end{array}\right]_{2}\left[\begin{array}{c}
n \\
2 j
\end{array}\right] .
\end{aligned}
$$

This is [A2, eq. (10.3)].

Proof. In the proof of Theorem 2.5, we can impose an upper bound on the largest part of $\lambda$, and take $q^{2 n}\left[\begin{array}{c}M+n \\ n\end{array}\right]_{2}$ for partitions into $n$ even parts, all $\leq 2 M$, in the first summand (instead of $q^{2 n} /\left(q^{2} ; q^{2}\right)_{n}$ ), and $q^{n}\left[\begin{array}{c}M+n-1 \\ n\end{array}\right]_{2}$ for partitions into $n$ odd parts, all $\leq 2 M-1$, in the second sum (instead of $\left.q^{n} /\left(q^{2} ; q^{2}\right)_{n}\right)$ in $(2.8)$.

Note that, in the first sum, $\widetilde{\lambda}_{1}=\lambda_{1}+2 j$ or $\widetilde{\lambda}_{1}=\lambda_{1}+2 j+1$, and in the second sum, $\widetilde{\lambda}_{1}=\lambda_{1}+2 j$ or $\widetilde{\lambda}_{1}=\lambda_{1}+2 j-1$ in 2.8 . Thus, taking $M \leftarrow M-j$ in the first sum and $M \leftarrow M-j+1$ in the second, we obtain the desired generating function.

Making the obvious changes in the above proofs, we can just as well obtain the following generating functions.

THEOREM 2.7 .

$$
\begin{aligned}
F_{o}(\infty)= & \sum_{j, n \geq 0} \frac{x^{n} y^{2 j+1} q^{2 j(2 j+1) / 2+n}}{\left(q^{2} ; q^{2}\right)_{n}}\left[\begin{array}{c}
n \\
2 j+1
\end{array}\right] \\
& +\sum_{j, n \geq 0} \frac{x^{n} y^{2 j} q^{(2 j-1) 2 j / 2+2 n}}{\left(q^{2} ; q^{2}\right)_{n}}\left[\begin{array}{c}
n \\
2 j
\end{array}\right] .
\end{aligned}
$$

This is [A2, eq. (10.16)].

THEOREM 2.8 .

$$
\begin{aligned}
F_{o}(2 M)= & \sum_{j, n \geq 0} x^{n} y^{2 j+1} q^{2 j(2 j+1) / 2+n}\left[\begin{array}{c}
M-j+n-1 \\
n
\end{array}\right]_{2}\left[\begin{array}{c}
n \\
2 j+1
\end{array}\right] \\
& +\sum_{j, n \geq 0} x^{n} y^{2 j} q^{(2 j-1) 2 j / 2+2 n}\left[\begin{array}{c}
M-j+n-1 \\
n
\end{array}\right]_{2}\left[\begin{array}{c}
n \\
2 j
\end{array}\right] .
\end{aligned}
$$

This is [A2, eq. (10.14)].

The example in $\$ 2.2$ can be reworked in this context with appropriate choices for $M$ and $j$. 
2.4. Upper parity indices in partitions with distinct parts. We recall two more generating functions from [A2]:

$$
\begin{aligned}
& D_{e}(N, y, x ; q):=D_{e}(N)=\sum_{r, m, n \geq 0} \delta_{e}(N, r, m, n) y^{r} x^{m} q^{n}, \\
& D_{o}(N, y, x ; q):=D_{o}(N)=\sum_{r, m, n \geq 0} \delta_{o}(N, r, m, n) y^{r} x^{m} q^{n},
\end{aligned}
$$

where $\delta_{e}(N, r, m, n)$ (respectively $\delta_{o}(N, r, m, n)$ ) is the number of partitions of $n$ into $m$ distinct parts each at most $N$ and with upper even (respectively odd) parity index equal to $r$ ([12, eqs. (9.1) and (9.2)]).

If we require distinct parts, then we modify the construction in the preceding section by taking a partition $\lambda$ into distinct parts, all odd or all even. Then as described in $\$ 2.1(2.2)$, we subtract the parts of $\mu^{\prime}$, the conjugate of $\mu$, a partition into distinct parts, all $<n$ (but not $\leq n$ ). Then we have the following generating functions.

THEOREM 2.9 .

$$
\begin{aligned}
D_{e}(\infty)= & \sum_{j, n \geq 0} \frac{x^{n} y^{2 j+1} q^{-2 j(2 j+1) / 2+n(n+1)}}{\left(q^{2} ; q^{2}\right)_{n}}\left[\begin{array}{c}
n \\
2 j+1
\end{array}\right]_{(-1)} \\
& +\sum_{j, n \geq 0} \frac{x^{n} y^{2 j} q^{-(2 j-1) 2 j / 2+n^{2}}}{\left(q^{2} ; q^{2}\right)_{n}}\left[\begin{array}{c}
n \\
2 j
\end{array}\right]_{(-1)} .
\end{aligned}
$$

Here, $\left[\begin{array}{l}A \\ B\end{array}\right]_{(-1)}$ is $\left[\begin{array}{l}A \\ B\end{array}\right]$ with $q$ replaced by $1 / q$, since we are subtracting parts of $\mu^{\prime}$. This is [A2, eq. (9.16)].

Again, we can impose some bound on the parts, and we can replace $q^{n(n+1)} /\left(q^{2} ; q^{2}\right)_{n}$ by $q^{n(n+1)}\left[\begin{array}{c}M \\ n\end{array}\right]_{2}$, which gives us partitions into $n$ distinct even parts $\leq 2 M$ in the first sum, and $q^{n^{2}} /\left(q^{2} ; q^{2}\right)_{n}$ by $q^{n^{2}}\left[\begin{array}{c}M \\ n\end{array}\right]_{2}$, which yields partitions into $n$ distinct odd parts $\leq 2 M-1$ in the second sum in (2.12). Since we are subtracting $2 j$ or $2 j+1$ from $\lambda_{1}$ in the first sum, and $2 j$ or $2 j-1$ in the second, we substitute $M \leftarrow M+j$ in both the first and the second sum. Thus, we obtain

THEOREM 2.10 .

$$
\begin{aligned}
D_{e}(2 M)= & \sum_{j, n \geq 0} x^{n} y^{2 j+1} q^{-2 j(2 j+1) / 2+n(n+1)}\left[\begin{array}{c}
M+j \\
n
\end{array}\right]_{2}\left[\begin{array}{c}
n \\
2 j+1
\end{array}\right]_{(-1)} \\
& +\sum_{j, n \geq 0} x^{n} y^{2 j} q^{-(2 j-1) 2 j / 2+n^{2}}\left[\begin{array}{c}
M+j \\
n
\end{array}\right]_{2}\left[\begin{array}{c}
n \\
2 j
\end{array}\right]_{(-1)} .
\end{aligned}
$$

Finally, we can repeat the arguments with the appropriate changes to obtain 
THEOREM 2.11 .

$$
\begin{aligned}
D_{o}(\infty)= & \sum_{j, n \geq 0} \frac{x^{n} y^{2 j+1} q^{-2 j(2 j+1) / 2+n^{2}}}{\left(q^{2} ; q^{2}\right)_{n}}\left[\begin{array}{c}
n \\
2 j+1
\end{array}\right]_{(-1)} \\
& +\sum_{j, n \geq 0} \frac{x^{n} y^{2 j} q^{-(2 j-1) 2 j / 2+n(n+1)}}{\left(q^{2} ; q^{2}\right)_{n}}\left[\begin{array}{c}
n \\
2 j
\end{array}\right]_{(-1)} .
\end{aligned}
$$

This is [A2, eq. (9.15)], and the generating function of partitions into distinct parts $\leq 2 M+1$ is as follows:

THEOREM 2.12 .

$$
\begin{aligned}
D_{o}(2 M+1) & \\
= & \sum_{j, n \geq 0} x^{n} y^{2 j+1} q^{-2 j(2 j+1) / 2+n^{2}}\left[\begin{array}{c}
M+j \\
n
\end{array}\right]_{2}\left[\begin{array}{c}
n \\
2 j+1
\end{array}\right]_{(-1)} \\
& +\sum_{j, n \geq 0} x^{n} y^{2 j} q^{-(2 j-1) 2 j / 2+n(n+1)}\left[\begin{array}{c}
M+j \\
n
\end{array}\right]_{2}\left[\begin{array}{c}
n \\
2 j
\end{array}\right]_{(-1)} .
\end{aligned}
$$

Noting that $\left[\begin{array}{l}A \\ B\end{array}\right]_{(-1)}=q^{-B(A-B)}\left[\begin{array}{l}A \\ B\end{array}\right]$, we get $[\mathbf{A 2}$, eqs. (9.4) and (9.3)] from (2.13) and (2.15), respectively.

The example in $\$ 2.1$ can be reworked in this context with appropriate choices for $M$ and $j$.

\section{Sieves}

3.1. Lower odd parity index in partitions with distinct parts. Here we begin by explaining [A2, eq. (7.5)].

THEOREM 3.1.

$$
P_{o}(y, x ; q)=(-x q)_{\infty} \sum_{n \geq 0} \frac{(y)_{n}(-x q)^{n}}{\left(q^{2} ; q^{2}\right)_{n}} .
$$

Proof. The factor $(-x q)^{n} /\left(q^{2} ; q^{2}\right)_{n}$ in the term under the sum generates exactly $n$ odd numbers $\lambda=\left(\lambda_{1}, \ldots, \lambda_{n}\right)$, not necessarily distinct, all having factor -1 . $(y q)_{n-1}$ generates a partition $\mu$ having distinct parts $<n$. Each factor in $(y q)_{n-1}$, say $\left(1-y q^{j}\right)$ for $0<j<n$, either leaves the -1 weighted numbers intact (1), or adds 1 to the first $j$ parts (another description of the method in $\$ 2.2$, creating a parity change exactly at the $j$ th part $\lambda_{j}$ (that is, $\left.\lambda_{j} \not \equiv \lambda_{j+1}(\bmod 2)\right)$, and commutes the factor -1 of $\lambda_{j}$ to $y\left(-y q^{j}\right)$. The remaining factor $(1-y)$ either leaves the smallest part $\lambda_{n}$ (which is odd) as it is, or commutes its factor from -1 to $y$. This way, we form $\tilde{\lambda}$ by adding all terms of $\mu^{\prime}$. If $-y$ is chosen in $(1-y)$, then the exponent $y$ accounts for the lower odd parity index in $\widetilde{\lambda}$, otherwise it gives one less than the lower 
odd parity index of $\tilde{\lambda}$. And remember that a part $\widetilde{\lambda}_{j}$ in $\tilde{\lambda}$ has factor $y$ if $\widetilde{\lambda}_{j} \not \equiv \widetilde{\lambda}_{j-1}(\bmod 2),-1$ otherwise for $j=2, \ldots, n$. Also, $\widetilde{\lambda}_{1}$ has factor either -1 or $y$. Finally, $(-x q)_{\infty}$ provides distinct terms, all having weight 1 , and provides no $y$ 's. Note that for any partition generated by the right hand side, the exponent of $y$ can never exceed the lower odd parity index.

Let $\beta=\left(\beta_{1}, \ldots, \beta_{m}\right)$ be a partition generated by the right hand side, along with some factor of $y$ to some power, which, as a matter of fact, is smaller than or equal to the lower odd parity index of $\beta$. It is clear that if a part is repeated in $\beta$, then one and only one of those parts could have come from $(-x q)_{\infty}$. We agree that it is the one with the largest index, namely the last of the occurrences.

When $\beta_{i}=\beta_{i-1} \neq \beta_{i+1}$ for some $i$ between 2 and $m\left(\beta_{m+1}=0\right)$, then there seem to be two possibilities. If $\beta_{i}$ does not have factor $y$, then $\beta_{i}$ is either contributed by $\left(1+x q^{i}\right)$ with weight 1 , or it is contributed by a term under the sum, with weight -1 . Thus, for any composition of $\beta$ without $\beta_{i}, \beta_{i}$ is introduced both with a plus and a minus sign. Else if $\beta_{i}$ has a factor $y$, then $\beta_{i-1}$ cannot assume a factor $y$. By the construction above, a factor $y$ is not possible for adjacent parts without a parity change. Also, by our agreement, $\beta_{i-1}$ could not have been contributed by $(-x q)_{\infty}$, which forces a parity change $\beta_{i-1} \not \equiv \beta_{i}(\bmod 2)$ to justify the $y$ factor for $\beta_{i}$, an impossibility. Therefore, $\beta$ 's with repetitions of parts are annihilated by the sieve on the right hand side.

We give an example on the fly to clarify the part of the proof above.

ExAmple. Let $\lambda=\left(7_{(-)}, 3_{(-)}, 3_{(-)}\right)$be given by $(-x q)^{3} /\left(q^{2} ; q^{2}\right)_{3}$, where subscripts indicate the factors -1 . Assume that $-y$ is chosen in $(1-y)$, and $\mu=1$ is given. Then $\widetilde{\lambda}=\left(8_{y}, 3_{(-)}, 3_{y}\right)$. Moreover, assume that we take 1 from the whole factor $(-x q)_{\infty}$, that is, no contribution from the first factor in the generating function, so that $\beta=\widetilde{\lambda}$. On the other hand, given $\lambda=\left(7_{(-)}, 3_{(-)}\right)$ by $(-x q)^{2} /\left(q^{2} ; q^{2}\right)_{2},-y$ from $(1-y)$, and $\mu=1$, so that $\widetilde{\lambda}=\left(8_{(y)}, 3_{(y)}\right)$ and 3 in $\left(1+x q^{3}\right)$ from $(-x q)_{\infty}$, we obtain $\beta=\left(8_{y}, 3_{(+)}, 3_{y}\right)$. Therefore, $\beta$ along with $y^{2}$ is generated both by a plus and a minus sign. Although $\beta$ has lower odd parity index 2 , it is annihilated.

When $\beta$ has distinct parts, along with some power of $y$, for a $\beta_{i}$ having a factor of $y$, if there are parts $\beta_{i-1}, \beta_{i-2}, \ldots, \beta_{i-s}$ none having a factor of $y$, such that $\beta_{i}$ and $\beta_{i-s}$ have the same parity, then $\beta_{i-s}$ could have come from $(-x q)_{\infty}$ with a plus sign, or from a term in the sum with minus sign. Such $\beta$ 's are annihilated by the sieve on the right hand side. This leaves us $\beta$ 's along with some power of $y$, whose single factors $y$ are given to $\beta_{i}$ 's for which $\beta_{i-1}$ has opposite parity, and among the parts greater than the largest $\beta_{i}$ to receive factor $y$, there are none with the same parity as $\beta_{i}$. 
If the exponent of $y$ is strictly less than the lower odd parity index of $\beta$, then there are exactly two distributions of $y$ 's. In one, $\beta_{1}$ receives $y$, and in the other $\beta_{i}$ receives $y, \beta_{1}, \ldots, \beta_{i-1}$ receive none. In the latter case, $\beta_{1}, \ldots, \beta_{i-1}$ have opposite parity to $\beta_{i}$. Now, in exactly one of the cases, the smallest part to receive a $y$ is even, and in the other, it is odd. If it is odd, then $\beta$ has no parts with factor -1 , hence it is counted as 1 . Else if it is even, then by construction, at least one odd part smaller than the smallest even part with a factor $y$ has factor -1 , forcing $\beta$ to be counted -1 . The above mentioned odd part exists, as discussed above, to receive 1 from the factor $(1-y)$ in the sum. In total, $\beta$ is annihilated.

Therefore, only $\beta$ 's accompanied by a power of $y$ equal to the lower odd parity index survive, and counted as 1 . This concludes the proof.

ExAmple. Given $\beta=(11,10,8,7,5,4,3)$ along with $y^{3}$, we need to give the factors $y$ to parts that alternate in parity in increasing order. Suppose that we chose

$$
\beta=\left(11,\{10\}, 8_{y}, 7,5_{y}, 4_{y}, 3\right),
$$

but we did not indicate the source of the other parts, which can be either $(-x q)_{\infty}$, or a term inside the sum, without factors $y$. Note that the 10 in curly braces can be generated as $10_{(-)}$or $10_{(+)}$. So that no matter what the signs of the other parts are, $\beta$ is generated with plus sign as well as minus sign the same number of times. This is the case whenever the smaller of a pair of parts of the same parity has factor $y$, and there are no other $y$ 's in between, hence no $y$ for the larger part. Thus, there are only two distributions of $y$ 's for this $\beta$. The first one is

$$
\beta=\left(11_{y}, 10_{y}, 8,7_{y}, 5,4,3\right) .
$$

For this possibility, 8 cannot be generated by a minus factor, since in that case 10 could not have received a $y$ by construction. $\left(\ldots, 10_{y}, 8_{(-)}, \ldots\right)$ in $\beta$ means that 10 and 8 are adjacent parts without parity change in between, so that the larger part $10_{y}$ having factor $y$ is not possible in the first place. A similar reasoning applies for 5 and 3 , so that all 8,5 and 3 have plus factors. For 4, it cannot have a minus factor because in that case it would be the smallest number in $\widetilde{\lambda}$, the part of $\beta$ coming from a term inside the sum. However, that smallest term with a minus factor can only be an odd number by construction. Therefore, $\beta$ as such is generated exactly once with plus sign.

The second possibility is

$$
\beta=\left(11,10_{y}, 8,7_{y}, 5,4_{y}, 3\right) .
$$

As discussed above, 8 and 5 have plus factors. 11 on the other hand has a plus sign for a different reason. Were 11 contributed by a term under the sum, i.e. if $\beta=\left(11_{(-)}, 10_{y}\right)$, we should have a factor $y$ for 11 , thanks to the 
parity change in between. On the other hand, if the smallest part to receive a $y$ is an even number, then there must be a smaller odd number, which is the smallest part of $\widetilde{\lambda}$, which evidently has a minus factor. So, 3 here has a minus factor. $\beta$ here is generated once by a minus sign, therefore it is finally annihilated.

However, if $\beta$ is accompanied by $y^{5}$, then the only surviving distribution of $y$ 's would be

$$
\beta=\left(11_{y}, 10_{y}, 8,7_{y}, 5,4_{y}, 3_{y}\right),
$$

which is generated exactly once with plus sign. Observe that the lower odd parity index of $\beta$ is 5 .

3.2. Lower odd parity index in unrestricted partitions. Here we explain a variant of [A2, eq. (8.4)].

THEOREM 3.2 .

$$
U_{o}(y, x ; q)=\frac{1}{(x q)_{\infty}} \sum_{n \geq 0} \frac{(-x)^{n} q^{n^{2}}(y ; 1 / q)_{n}}{\left(q^{2} ; q^{2}\right)_{n}} .
$$

This is converted back to [A2, eq. (8.4)] by changing the base to $q$ in $(y ; 1 / q)_{n}$ [GR, (1.2.24)].

Proof. The factor $(-x)^{n} q^{n^{2}} /\left(q^{2} ; q^{2}\right)_{n}$ under the sum generates partitions $\lambda$ into distinct odd parts, each having factor -1 . The factor $(y / q ; 1 / q)$ generates partitions $\mu$ into distinct parts $<n$. We form $\tilde{\lambda}$ by subtracting parts of $\mu^{\prime}$ from parts of $\lambda$ as described in 3.1 with the following specifications. A factor in $(y / q ; 1 / q)$, say $\left(1-y / q^{i}\right)$ for $0<i<n$, either leaves the partition intact (1), or subtracts 1 from the first $j$ parts introducing a parity change at $\lambda_{j}$, namely $\lambda_{j} \not \equiv \lambda_{j+1}(\bmod 2)$, and commutes the factor of $\lambda_{j}$ from -1 to $y\left(-y / q^{i}\right) .(1-y)$ either leaves the smallest part as it is, or changes its factor from -1 to $y$. So that the exponent of $y$ accompanying $\tilde{\lambda}$ gives us either the lower parity index of $\widetilde{\lambda}$, or one less than that, depending on the factor of the smallest part $\lambda_{n}$, which is odd. Finally, $1 /(x q)_{\infty}$ brings unrestricted partitions, all parts having factor 1 .

Let $\beta=\left(\beta_{1}, \ldots, \beta_{m}\right)$ be a partition generated by the right hand side, along with a factor of $y$ whose exponent is at most the lower odd parity index of $\beta$.

We can pick at least as many distinct parts of $\beta$ as the exponent of $y$ that alternate in parity, and argue that those parts come from the sum, each having factor -1 or $y$. This also introduces a distribution of $y$ 's among parts of $\beta$. We agree that the first part to appear among equal parts (the one with the smallest index) can possibly have come from the infinite sum, to fix an order of parts coming from the factor $1 /(x q)_{\infty}$ or from the sum. 
The argument from 3.1 then applies almost word for word, except that $\beta$ 's are not required to have distinct parts. For a $\beta_{i}$ having a factor of $y$, if there are parts $\beta_{i-1}, \beta_{i-2}, \ldots, \beta_{i-s}$, none having a factor of $y$, such that $\beta_{i} \neq \beta_{i-s}$ have the same parity, $\beta_{i-s}$ could have come from $1 /(x q)_{\infty}$ with a plus sign, or from a term in the sum with minus sign. Such $\beta$ 's are annihilated by the sieve on the right hand side. This leaves us $\beta$ 's along with some power of $y$, whose single factors $y$ are given to $\beta_{i}$ 's for which $\beta_{i-1}$ has opposite parity, and among the parts greater than the largest $\beta_{i}$ to receive factor $y$, there are none with the same parity as $\beta_{i}$.

If the exponent of $y$ is strictly less than the lower odd parity index of $\beta$, then there are exactly two distributions of $y$ 's. In one, $\beta_{1}$ receives $y$, and in the other $\beta_{i}$ receives $y, \beta_{1}, \ldots, \beta_{i-1}$ receive none. In the latter case, $\beta_{1}, \ldots, \beta_{i-1}$ have opposite parity to $\beta_{i}$. Now, in exactly one of the cases, the smallest part to receive a $y$ is even, and in the other, it is odd. If it is odd, then $\beta$ has no parts with factor -1 , hence it is counted as 1 . Else if it is even, then by construction at least one odd part smaller than the smallest even part with a factor $y$ has factor -1 , forcing $\beta$ to be counted -1 . In total, $\beta$ is annihilated.

Therefore, only $\beta$ 's accompanied by a power of $y$ equal to the lower odd parity index survive and are counted as 1 . This establishes the sieve.

EXAMPLE. We return to $\beta=(11,10,8,7,5,4,3)$ from the preceding section, this time accompanied by $y^{1}=y$. Again, most of the alternatives to place $y$ vanish in the sieve, and two remain to discuss. The first one is

$$
\beta=\left(11_{y}, 10,8,7,5,4,3\right) .
$$

Here, no part can assume a minus factor, so $\beta$ as such is generated once with plus sign. The second one is

$$
\beta=\left(11,10_{y}, 8,7,5,4,3\right) .
$$

Since the smallest part to receive factor $y$ is even, there is at least one smaller odd part (there are three). At least one of them must receive a minus factor. The possibilities are

$$
\begin{array}{ll}
7_{(-)}, 5_{(-)}, 3_{(-)}, & \text {counted as }-; \\
7_{(-)}, 5_{(-)}, 3_{(+)}, & \text {counted as }+; \\
7_{(-)}, 5_{(+)}, 3_{(-)}, & \text {counted as }+; \\
7_{(-)}, 5_{(+)}, 3_{(+)}, & \text {counted as }-; \\
7_{(+)}, 5_{(-)}, 3_{(-)}, & \text {counted as }+; \\
7_{(+)}, 5_{(-)}, 3_{(+)}, & \text {counted as }-; \\
7_{(+)}, 5_{(+)}, 3_{(-)}, & \text {counted as }-.
\end{array}
$$


Thus, $\beta$ is generated exactly once with a minus sign when 10 receives a minus factor. Together with the former option, $\beta$ is annihilated.

Note that exactly one among equal parts could receive factor $y$ or $(-)$. All others come with $(+)$ sign. Hence, the discussion would be just the same for $\beta_{0}=(11,11,11,10,8,8,7,5,5,5,5,4,3,3)$ along with $y^{1}=y$.

We remark that there is a similar way to prove [A2, eq. (8.4)] in the form it is given:

$$
U_{o}(y, x ; q)=\frac{1}{(x q)_{\infty}} \sum_{n \geq 0} \frac{x^{n} y^{n} q^{n(n+1) / 2}(1 / y)_{n}}{\left(q^{2} ; q^{2}\right)_{n}} .
$$

Here, $x^{n} y^{n} q^{n(n+1) / 2} /\left(q^{2} ; q^{2}\right)_{n}$ generates a partition with full lower parity index, and we interpret $(1 / y)_{n}$ as ruling out parity changes, and replacing $y$ 's with -1 's. This method is a bit more involved. The argument developed above seems to be more instructive.

3.3. An unexpected relation. Here we prove [A2, eq. (8.13)].

THEOREM 3.3 .

$$
U_{o}(y, x ; q)=\frac{1}{(x q)_{\infty}} P_{o}(-y q,-x / q ; q) .
$$

Proof. Given a partition $\lambda=\left(\lambda_{1}, \ldots, \lambda_{n}\right)$ enumerated by $P_{o}(y, x ; q)$, assume that the factors $y$ are assigned to $\lambda_{i}$ 's for which $\lambda_{i+1}$ has opposite parity. Now, replacing $y$ by $-y q$ and $x$ by $-x / q$ subtracts 1 from the parts having no factor $y$ and gives them factors -1 . The parts having factor $y$ are intact. Call the transformed partition $\widetilde{\lambda}$. If the smallest part is 1 , it had the factor $y$, so that $\widetilde{\lambda}$ has $n$ distinct parts.

The exponent of $y$ is equal to the lower odd parity index of $\tilde{\lambda}$, or one less than that. To see this, note that the parts without factor $y$ that fall between two parts with factor $y$ are all of the same parity as the smaller end before the decrease, and of the same parity as the larger end afterwards. The parts $\lambda_{1}, \ldots, \lambda_{s-1}$ without a factor $y$ followed by $\lambda_{s}$ with factor $y$ must all be of the same parity as $\lambda_{s}$. Hence a parity change occurs after the decrease which is not accounted for by a $y$ unless $\lambda_{1}$ and $\lambda_{2}$ are of opposite parities. This is the place where the lower parity index of $\widetilde{\lambda}$ increases by one. The parts $\lambda_{n}, \ldots, \lambda_{n-r+1}$ without factors $y$ must all be even while $\lambda_{n-r}$ with factor $y$ must be odd. After the decrease, $\widetilde{\lambda}_{n}, \ldots, \widetilde{\lambda}_{n-r+1}$ become odd with $\widetilde{\lambda}_{n-r}$ having factor $y$ also odd. If all parts are even, then the two previous cases coincide, and the lower odd parity index increases by one. Note that, unlike in $\$ 3.1$ and $3.2, \widetilde{\lambda}_{i}$ assumes a factor $y$ precisely when $\widetilde{\lambda}_{i-1}$ is of the opposite parity for $i$ between 2 and $n$, and $\widetilde{\lambda}_{1}$ has a factor $y$ when $\widetilde{\lambda}_{2}$ and $\widetilde{\lambda}_{1}$ are of opposite parities and $\widetilde{\lambda}_{2}$ has factor $y$, in which case $\lambda_{1}$ had a $y$ to begin with. Otherwise $\widetilde{\lambda}_{1}$ may have either $y$ or $(-)$. All other parts have minus factors. 
Finally, $\widetilde{\lambda}$ is augmented by $1 /(x q)_{\infty}$, which brings unrestricted partitions. Among the parts that are equal, we agree that the last occurrence (the one with the largest index) could have come from the infinite sum, and the following occurrences come from the infinite product; or else all occurrences come from the infinite product. Note that exactly one among the same parts is subject to having factor $y$ or $(-)$.

Let $\beta=\left(\beta_{1}, \ldots, \beta_{m}\right)$ be a partition given by the right hand side along with some power of $y$. By the remark in the above paragraph, it suffices to pick one instance of repeated parts in $\beta$. So, without loss of generality we may assume that $\beta$ has distinct parts. It is immediate that the lower odd parity index of $\beta$ is at least as large as the exponent of $y$. Unlike in 3.1 and 3.2 , the smallest part to receive $y$ must be odd by construction.

We employ a slightly different sieve here. Whenever $\beta$ is given along with some power of $y$, there are two cases where $\beta$ is annihilated. First, given a distribution of $y$ 's, for a $\beta_{i}$ having factor $y$, there is $\beta_{i+s}$ of the same parity without factor $y$, and $\beta_{i+1}, \ldots, \beta_{i+s-1}$ have no $y$ 's either. In this case, $\beta_{i+s}$ could have come from the second factor on the right hand side of $(3.3)$, hence with a minus sign, or from the first factor with a plus sign. Or else, for the smallest $\beta_{i}$ to receive a factor $y$, there is a smaller $\beta_{i-s}$ of the opposite parity. In this case, $\beta_{i-s}$ could have been the largest part $\widetilde{\lambda}_{1}$ with the minus factor as discussed above, or $\beta_{i-s}$ could have been contributed by $1 /(x q)_{\infty}$, hence having plus sign. In either option, $\beta$ is annihilated.

It is evident that when the accompanying power of $y$ has exponent strictly less than the lower odd parity index of $\beta$, we fall into one of the cases above, thus $\beta$ is annihilated. Also, for that exponent equal to the lower odd parity index of $\beta$, there is a unique distribution of $y$ 's so as to avoid the former case above, which assigns a plus sign to $\beta$. In other words, $\beta$ survives along with a power of $y$ the exponent of which equals the lower even parity index, and when the $y$ 's are assigned to $\beta_{i}$ 's for which $\beta_{i+1}$ is of the opposite parity $\left(\beta_{m+1}=0\right)$.

EXAmple. We continue with the same $\beta=(11,10,8,7,5,4,3)$ along with $y^{3}$. For the following distribution of $y$ 's:

$$
\beta=\left(11,10,8,7,5_{y}, 4_{y}, 3_{y}\right),
$$

both 8 and 10 may be contributed as parts of $\widetilde{\lambda}$, or parts from $1 /(x q)_{\infty}$. Hence both may have plus or minus factors. $\beta$ as such is annihilated. Other distributions may be considered, but all will be generated with minus signs as many times as they are generated with plus signs.

For $\beta=(11,10,8,7,5,4,3)$ along with $y^{5}$ (note $\beta$ has lower odd parity index 5), we consider the surviving distribution of $y$ 's in $\$ 3.1$ and $\$ 3.2$.

$$
\beta=\left(11_{y}, 10_{y}, 8,7_{y}, 5,4_{y}, 3_{y}\right) .
$$


Here, 8 has no $y$ 's, and it immediately follows 10 , which does have factor $y$. By the argument above, 8 could have been contributed as a part of $\widetilde{\lambda}$, hence having a minus factor, or by $1 /(x q)_{\infty}$ with plus sign, so $\beta$ as such is annihilated by the sieve in this section. The same applies for 5 following 7 . In case 8 and 5 are parts of $\widetilde{\lambda}$, we have no contribution at all from $1 /(x q)_{\infty}$, so that

$$
\widetilde{\lambda}=\left(11_{y}, 10_{y}, 8_{(-)}, 7_{y}, 5_{(-)}, 4_{y}, 3_{y}\right) .
$$

To get $\lambda$, we just remove the minus factors from the parts that have it, and add 1 to them, as described. Thus,

$$
\lambda=\left(11_{y}, 10_{y}, 9,7_{y}, 6,4_{y}, 3_{y}\right) .
$$

However, if we take

$$
\beta=\left(11_{y}, 10,8_{y}, 7,5_{y}, 4_{y}, 3_{y}\right),
$$

we fall into neither case discussed, so neither 10 nor 7 could have been generated with minus signs. In this case, the parts 10 and 7 come from $1 /(x q)_{\infty}$.

4. Partitions with ample part size. We recall another definition from [A2].

Definition 4.1. A partition $\lambda$ is said to have even (resp. odd) ample part size if each part $\lambda_{i}$ is larger than the upper even (resp. odd) parity index of $\lambda$.

THEOREM 4.2 .

$$
\varrho_{e}(y, x ; q)=\sum_{n \geq 0} \frac{x^{n} q^{n^{2}}(-y q ; q)_{n}}{\left(q^{2} ; q^{2}\right)_{n}}
$$

generates partitions into distinct parts with even ample part size, where the exponent of $x$ keeps track of the number of parts, and the exponent of $y$ the upper even parity index.

Proof. Now, $x^{n} q^{n^{2}} /\left(q^{2} ; q^{2}\right)_{n}$ generates partitions $\lambda=\left(\lambda_{1}, \ldots, \lambda_{n}\right)$ with distinct odd parts. Originally, the upper even index is zero, there is nothing to prove. For each factor $\left(1+y q^{i}\right), 1 \leq i \leq n$, we add 1 to parts $\lambda_{n}, \lambda_{n-1}, \ldots, \lambda_{n-(i-1)}$, so that the parts of $\lambda$ are still distinct, but there is a parity change $\lambda_{i} \not \equiv \lambda_{i-1}(\bmod 2)$. Observe that if $\lambda_{i_{0}}$ is the largest part to be altered, then it becomes even. So that the exponent of $y$ indeed counts the upper even parity index. Also, each parity change or each introduction of a factor $y$ adds 1 to $\lambda_{n} \geq 1$. Therefore, $\widetilde{\lambda}_{n}$, the transformed $\lambda_{n}$, is always greater than the upper even parity index, hence so are the other parts. 
Conversely, given a partition into distinct parts with even ample part size, we can subtract 1s from sufficiently many smallest parts from the partition $\widetilde{\lambda}$ for each parity change, so as to get $\lambda$, a partition into distinct odd numbers. At each step, we recover a $y q^{i}$. The procedure is clearly reversible.

ExAmple. In $\S 2.1, \widetilde{\lambda}=(15,13,12,8,5,4)$ has upper even parity index 3 , and all parts are greater than 3 , so that $\tilde{\lambda}$ has ample part size. The picture is

$$
\widetilde{\lambda}=(15,13, \underbrace{12,8}_{1 \text { added }}, \underbrace{5}_{2 \text { added }}, \underbrace{4}_{\text {added }})
$$

so that

$$
\lambda=(15,13,11,7,3,1),
$$

a partition into distinct odd parts, and $\mu^{\prime}=(3,2,1,1)$ (hence $\mu=(4,2,1)$ ), as claimed.

Note that upper even parity index is even when the smallest part is odd, and it is odd when the smallest part is even. This shows that the inequality is strict in the definition. This fact has been communicated by Andrews to the author, but it is not explicitly included in A2.

In the above argument, if we replace $x^{n} q^{n^{2}} /\left(q^{2} ; q^{2}\right)_{n}$ by $\left[\begin{array}{l}N \\ n\end{array}\right]_{2} q^{n^{2}} x^{n}$, then we get $\lambda=\left(\lambda_{1}, \ldots, \lambda_{n}\right)$ as a partition into distinct odd parts $\leq 2 N-1$. So that $\widetilde{\lambda}_{1} \leq 2 N$, since $\lambda_{1} \leq 2 N-1$, and it may be added at most 1 , coming from the factor $\left(1+y q^{n}\right)$. This yields

THEOREM 4.3.

$$
\rho_{e}(N, y, x ; q)=\sum_{n=0}^{N}\left[\begin{array}{l}
N \\
n
\end{array}\right]_{2} q^{n^{2}} x^{n}(-y q ; q)_{n}
$$

generates partitions into distinct parts with even ample part size, each at most $2 N$, where the exponent of $x$ keeps track of the number of parts, and the exponent of $y$ the upper even parity index.

This is [A2, eq. (11.2)].

If we begin with partitions into distinct even parts, and interpret the factor $(-y q ; q)_{n}$ as above, we get the generating function for partitions into distinct parts with odd ample part size, and its variant where parts are bounded. Here, there are instances where the equality can hold in the definition, so we emphasize that the inequality is strict. For instance, the only partition of 1 is not counted as having odd ample part size. As argued in [A2], this is equivalent to replacing $x$ by $x q$ in the above generating functions.

Thus, we obtain: 
THEOREM 4.4 .

$$
\varrho_{o}(y, x ; q)=\sum_{n \geq 0} \frac{x^{n} q^{n(n+1)}(-y q ; q)_{n}}{\left(q^{2} ; q^{2}\right)_{n}}
$$

generates partitions into distinct parts with odd ample part size.

THEOREM 4.5.

$$
\rho_{o}(N, y, x ; q)=\sum_{n=0}^{N}\left[\begin{array}{l}
N \\
n
\end{array}\right]_{2} q^{n(n+1)} x^{n}(-y q ; q)_{n}
$$

generates partitions into distinct parts $\leq 2 N+1$ with odd ample part size.

In both (4.3) and (4.4), the exponent of $x$ keeps track of the number of parts, and the exponent of $y$ the upper odd parity index.

\section{References}

[A1] G. E. Andrews, The Theory of Partitions, Addison-Wesley, Reading, MA, 1976; Reissued: Cambridge Univ. Press, 1998.

[A2] -, Parity in partition identities, Ramanujan J., to appear.

[GR] G. Gasper and M. Rahman, Basic Hypergeometric Series, 2nd ed., Encyclopedia Math. Appl. 96, Cambridge Univ. Press, 2004.

Kağan Kurşungöz

Department of Mathematics

The Pennsylvania State University

University Park, PA 16802, U.S.A.

E-mail: kursun@math.psu.edu

Received on 30.1.2009

and in revised form on 20.1.2010 